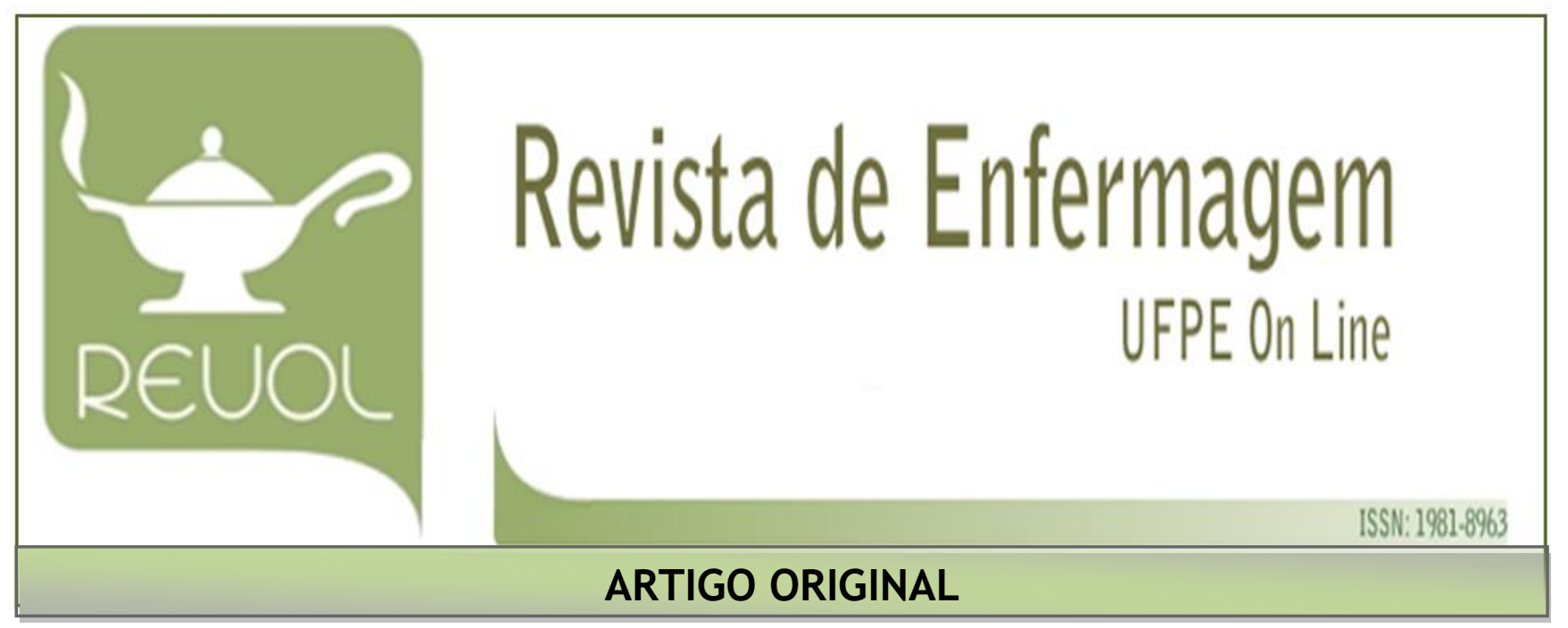

\title{
ACESSIBILIDADE AO TRATAMENTO E ESTADO DE SAÚDE DE PACIENTES HEMODIALÍTICOS
}

\section{ACCESSIBILITY TO TREATMENT AND HEALTH STATUS OF HEMODIALYTIC PATIENTS ACCESIBILIDAD AL TRATAMIENTO Y ESTADO DE SALUD DE PACIENTES HEMODIALÍTICOS}

Dandara Novakowski Spigolon ${ }^{1}$, Elen Ferraz Teston ${ }^{2}$, Maria Antonia Ramos Costa ${ }^{3}$, Edilaine Maran ${ }^{4}$, Rebeca Rosa de Souza ${ }^{5}$, Antônio Moreira Neto ${ }^{6}$

\begin{abstract}
RESUMO
Objetivo: identificar a acessibilidade ao tratamento e o estado de saúde de pacientes em hemodiálise. Método: estudo quantitativo, transversal, descritivo, realizado em uma clínica de nefrologia. Os dados foram coletados por meio de entrevista, analisados utilizando estatística descritiva e apresentados em tabelas de distribuição de frequência absoluta e relativa. Resultados: dos 151 pacientes, 49,6\% são idosos; 54,3\% têm baixa escolaridade; $66,2 \%$ possuem renda mensal de até dois salários mínimos; $93,4 \%$ têm seu tratamento financiando pelo Sistema Único de Saúde, porém, 45,7\% referem gastos com tratamento; 66,9\% mencionam a inobservância de tratamento conservador; $84,1 \%$ receberam cuidados pré-diálise; $84,1 \%$ declaram a autopercepção de saúde como boa e metade deles é acompanhada pela atenção primária à saúde (50,3\%). Conclusão: necessita-se do fortalecimento da rede de apoio social e de atenção à saúde, uma vez que a condição de vulnerabilidade caracterizada elucida novos desafios no desenvolvimento de ações na promoção à saúde, prevenção de complicações e na acessibilidade e organização do fluxo de atendimento. Descritores: Acesso aos Serviços de Saúde; Atenção Primária à Saúde; Assistência Integral à Saúde; Diálise Renal; Insuficiência Renal Crônica; Saúde Pública.
\end{abstract}

\section{ABSTRACT}

Objective: to identify accessibility to treatment and health status of hemodialysis patients. Method: quantitative, cross - sectional, descriptive study performed at a nephrology clinic. Data were collected through interviews, analyzed using descriptive statistics and presented in tables of absolute and relative frequency distribution. Results: of the 151 patients, $49.6 \%$ were elderly; $54.3 \%$ have low schooling; $66.2 \%$ have a monthly income of up to two minimum wages; $93.4 \%$ have their treatment financed by the Unified Health System, however, $45.7 \%$ refer to treatment expenses; $66.9 \%$ mention noncompliance with conservative treatment; $84.1 \%$ received pre-dialysis care; $84.1 \%$ declare their self-perception of health as good and half of them are accompanied by primary health care $(50.3 \%)$. Conclusion: it is necessary to strengthen the social support network and health care, since the condition of vulnerability characterized elucidates new challenges in the development of actions in health promotion, prevention of complications and in the accessibility and organization of the flow of care. Descritores: Health Services Accessibility; Primary Health Care; Comprehensive Health Care; Renal Dialysis; Renal Insufficiency Chronic; Public Health.

\section{RESUMEN}

Objetivo: identificar la accesibilidad al tratamiento y el estado de salud de los pacientes en hemodiálisis. Método: estudio cuantitativo, transversal, descriptivo, realizado en una clínica de nefrología. Los datos fueron recolectados por medio de encuestas, analizados utilizando estadística descriptiva y presentados en tablas de distribución de frecuencia absoluta y relativa. Resultados: de los 151 pacientes, el 49,6\% son ancianos; $54,3 \%$ tiene baja escolaridad; El $66,2 \%$ poseen renta mensuales de hasta dos salarios mínimos; $93,4 \%$ tiene su tratamiento financiando por el Sistema Único de Salud, pero el, 45,7\% refiere gastos con tratamiento; $66,9 \%$ mencionan la inobservancia de tratamiento conservador; el 84,1\% recibieron atención pre-diálisis; $\mathrm{El}$ $84,1 \%$ declaran la auto percepción de salud como buena; y la mitad de ellos son acompañados por la atención primaria a la salud $(50,3 \%)$. Conclusión: se requiere del fortalecimiento de la red de apoyo social y de atención a la salud, una vez que la condición de vulnerabilidad caracterizada elucida nuevos desafíos en el desarrollo de acciones en la promoción a la salud, prevención de complicaciones y en la accesibilidad y organización del flujo de atención. Descritores: Acceso a los Servicios de Salud; Atención Primaria a la Salud; Atención Integral de Salud; Diálisis Renal; Insuficiencia Renal Crónica; Salud Pública.

1,2,3Doutoras, Universidade Estadual do Paraná/UNESPAR. Paranavaí (PR), Brasil. E-mail: dandaraspigolon@gmail.com ORCID iD: https://orcid.org/0000-0002-9615-4420; E-mail: ferrazteston@gmail.com ORCID iD: https://orcid.org/0000-0002-1252-9708; E-mail: https://orcid.org/0000-0002-9615-4420; E-mail: ferrazteston@gmail.com ORCID iD: https://orcid.org/0000-0002-1252-9708; E-mail: Paranavaí (PR), Brasil. E-mail: edi_enf@hotmail.com ORCID iD: https://orcid.org/0000-0001-6085-2692; 5.6 Enfermeiras (egressas), Universidade Estadual do Paraná/UNESPAR. Paranavaí (PR), Brasil. E-mail: resouza15@hotmail.com ORCID iD: https://orcid.org/00000001-6501-3249; E-mail: neto_moreira@outlook.com ORCID iD: https://orcid.org/0000-0002-8556-618X 


\section{INTRODUÇÃO}

A doença renal crônica constitui-se em um importante problema de saúde pública em decorrência da elevada prevalência de morbidade e mortalidade. É caracterizada pela perda progressiva da função renal, por mais de três meses, na qual o organismo não mantém o equilíbrio metabólico e hidroeletrolítico. Quando progride para uma taxa de filtração glomerular $<15$ $\mathrm{mL} / \mathrm{min} / 1,73 \mathrm{~m}^{2}$ (estádio cinco dialítico), inicia-se regularmente uma terapia de substituição renal para a conservação da vida. $^{1}$

Para fins de organização do cuidado holístico ao paciente com doença renal crônica, o tratamento é classificado em conservador (estádios um a três), pré-diálise (estádios quatro e cinco ND - não dialítico) e terapia de substituição renal (estádio cinco Ddialítico). ${ }^{2} 0$ número de pacientes com doença renal crônica em estádio cinco dialítico - iniciando terapia de substituição renal - está aumentando no mundo todo, o que resulta em custos econômicos e sociais substanciais, consumindo orçamentos importantes de saúde em muitos países. ${ }^{3} 0$ número total estimado de pacientes em diálise foi de 112.004 e as estimativas nacionais no tratamento dialítico das taxas de prevalência são de 552 e de incidência de 180 pacientes por milhão da população global. ${ }^{4}$

Entre as terapias de substituição renal, a hemodiálise é o método de tratamento mais comum e o mais utilizado no Brasil, com prevalência de $91,4 \%$ em $2014 .^{3}$ Foram gastos, nos anos de 2008 e 2011, com procedimentos de hemodiálise no país, cerca de US\$ $723.841 .688,56$ e US\$ 970.354.599,98, respectivamente, sendo estimado um custo anual médio por paciente de US\$ 7.932,52, em 2008, e de US\$ 9.112,75, em 2011. ${ }^{5}$

Aliado ao impacto econômico, a doença renal crônica e a hemodiálise provocam uma sucessão de situações que afetam os aspectos físicos e psicológicos, além das repercussões pessoais, familiares e sociais do paciente. ${ }^{6-8}$ Nesse contexto, impõe-se uma rotina de tratamento que modifica o seu modo de viver impactando na percepção de saúde e manejo da doença. ${ }^{7-9}$

Atinente a isso, salienta-se a importância da acessibilidade ao tratamento conservador, pré-diálise e terapia de substituição renal e do cuidado ofertado pela equipe profissional de saúde durante esses diferentes segmentos de tratamentos. ${ }^{6}$ Durante a sessão de HD, o profissional da Enfermagem tem um papel importante, uma vez que envolve, além da execução de técnicas/procedimentos, o desenvolvimento de ações educativas que qualificam a assistência e estimulam 0 autocuidado desses pacientes no domicílio. ${ }^{6}$ Adicionalmente, o levantamento de características sociodemográficas e clínicas, desde a entrada do paciente até a saída da sessão de hemodiálise, permite 0 planejamento de cuidados individualizados e que atendam às reais necessidades desses indivíduos. ${ }^{6-7}$

Frente a esse cenário, se faz necessário o apoio das políticas públicas de saúde para que os profissionais, em especial o enfermeiro, que prestam estes cuidados, possam estimular as capacidades destes indivíduos contribuindo que se adaptem de maneira positiva ao novo estilo de vida, assumam o controle do seu tratamento e melhorem os resultados de saúde. ${ }^{9}$ Para tanto, torna-se essencial desvelar elementos influentes no processo assistencial ao doente renal crônico.

\section{OBJETIVO}

- Identificar a acessibilidade ao tratamento e o estado de saúde de pacientes em hemodiálise.

\section{MÉTODO}

Trata-se de um estudo quantitativo, transversal, descritivo, em uma clínica especializada em nefrologia que atende pacientes da região noroeste do Estado do Paraná (Sistema Único de Saúde e conveniados).

Elegeram-se como critérios de inclusão: pacientes com doença renal crônica em estádio cinco hemodialítico em tratamento na referida clínica, de ambos os sexos e com idade superior a 18 anos. Por sua vez, pacientes em tratamento psiquiátrico, com problemas cognitivos ou comprometimento de compreensão do estudo, mulheres grávidas e transplantados constituíram critérios de exclusão.

Inicialmente, havia 188 indivíduos cadastrados no local de estudo em hemodiálise. Destes, 13 foram excluídos (sete em tratamento psiquiátrico, com problemas cognitivos ou comprometimento de compreensão do estudo e três transplantados). Desse modo, o tamanho da amostra foi obtido por meio de cálculo amostral simples considerando os 175 indivíduos elegíveis, nível de significância (a) de 0,05 , poder estatístico do teste de $95 \%$ e possibilidade de perda amostral de $20 \%$, o que resultou em uma amostra de 156 indivíduos. Após o convite para participar do estudo, 
Spigolon DN, Teston EF, Costa MAR et al.

cinco indivíduos recusaram, o que resultou em uma amostra final de 151 sujeitos.

Coletaram-se os dados a partir da elaboração de um questionário semiestruturado pelos próprios autores com 20 questões que abordavam as características socioeconômicas, o financiamento, a acessibilidade e o estado de saúde. A coleta de dados ocorreu por meio de entrevista durante as sessões de hemodiálise, com duração média de 25 minutos, nos meses de julho e agosto de 2016. Para complementar as informações necessárias, os prontuários dos pacientes foram acessados posteriormente à entrevista. Após o registro dos dados em uma planilha no Microsoft Excel 2013, procedeu-se à análise por meio de estatística descritiva realizada no programa Statistical Package for the Social Sciences $\left(\right.$ SPSS) ${ }^{\circledR}$, versão 2.0, e apresentaram-se os resultados em tabelas de distribuição de frequência absoluta e relativa.
Acessibilidade ao tratamento e estado...

Aprovou-se o estudo no Comitê de Ética em Pesquisa sob o parecer $n^{\circ}$. 1.624.927, no ano de 2016, e este foi conduzido de acordo com as recomendações da Resolução $n^{\circ}$ 510, de 2016, do Conselho Nacional de Saúde/Ministério da Saúde.

\begin{tabular}{|l|}
\hline RESULTADOS \\
\hline \hline
\end{tabular}

Dos 151 pacientes da pesquisa, prevaleceram: $63,6 \%$ do sexo masculino; $45,7 \%$ brancos; $49,6 \%$ com mais de 60 anos de idade; $61,6 \%$ nasceram na região Sul; $55,6 \%$ são casados; $54,3 \%$ possuem o ensino fundamental incompleto; $66,2 \%$ ganham até dois salários mínimos; $43,7 \%$ estão aposentados e 35,8\% recebem auxílio-doença.

Na tabela 1, observam-se as características relacionadas ao financiamento e à acessibilidade ao tratamento e acompanhamento do paciente em hemodiálise.

Tabela 1. Financiamento e acessibilidade ao tratamento e acompanhamento do paciente em hemodiálise segundo o financiamento, transporte, tratamento conservador, tratamento pré-diálise e acompanhamento pela Unidade Básica de Saúde. Paranavaí (PR), Brasil, 2016.

\begin{tabular}{|c|c|c|}
\hline \multirow{2}{*}{ Variáveis } & \multicolumn{2}{|c|}{ Pacientes $(\mathrm{n}=151)$} \\
\hline & $\mathrm{N}$ & $\%$ \\
\hline \multicolumn{3}{|l|}{ Tratamento Financiado } \\
\hline Sistema Único de Saúde & 141 & 93,4 \\
\hline Convênios de Saúde & 10 & 6,6 \\
\hline \multicolumn{3}{|l|}{ Gastos Atuais Relacionados ao Tratamento da Doença } \\
\hline Não & 82 & 54,3 \\
\hline Sim & 69 & 45,7 \\
\hline Com Medicamentos & 63 & 41,7 \\
\hline Hospedagem e combustível & 6 & 4,0 \\
\hline \multicolumn{3}{|l|}{ Transporte para a clínica de diálise } \\
\hline Carro próprio & 36 & 23,8 \\
\hline Transporte fornecido pelo município & 110 & 72,8 \\
\hline Carona de terceiros & 3 & 2,0 \\
\hline Andando & 2 & 1,3 \\
\hline \multicolumn{3}{|l|}{$\begin{array}{l}\text { Este transporte permite acompanhante para as consultas e as práticas do } \\
\text { tratamento }\end{array}$} \\
\hline Não & 117 & 77,5 \\
\hline Às vezes & 8 & 5,3 \\
\hline Sim & 26 & 17,2 \\
\hline \multicolumn{3}{|l|}{ Tratamento Conservador } \\
\hline Não & 103 & 68,2 \\
\hline Sim & 48 & 31,8 \\
\hline \multicolumn{3}{|l|}{ Tratamento pré-diálise } \\
\hline Não & 24 & 15,9 \\
\hline Sim & 127 & 84,1 \\
\hline \multicolumn{3}{|l|}{$\begin{array}{l}\text { A Unidade Básica de Saúde de referência faz acompanhamento do seu } \\
\text { tratamento }\end{array}$} \\
\hline Não & 32 & 21,2 \\
\hline Sim & 76 & 50,3 \\
\hline Às vezes & 43 & 28,5 \\
\hline
\end{tabular}

Com relação ao tempo de hemodiálise, $82 \%$ dos indivíduos referiram ter iniciado há mais de um ano. A tabela 2 apresenta características relacionadas à percepção do indivíduo quanto ao estado de saúde e efeitos do tratamento dialítico. 
Tabela 2. Percepção do próprio estado de saúde e tratamento dialítico segundo a autopercepção de saúde, a dor e os efeitos da doença renal crônica. Paranavaí (PR), Brasil, 2016.

\begin{tabular}{|c|c|c|}
\hline \multirow{2}{*}{ Variáveis } & \multicolumn{2}{|c|}{ Pacientes $(n=151)$} \\
\hline & $\mathrm{N}$ & $\%$ \\
\hline \multicolumn{3}{|l|}{ Autopercepção de saúde } \\
\hline Boa & 127 & 84,1 \\
\hline Ruim & 24 & 15,9 \\
\hline \multicolumn{3}{|c|}{$\begin{array}{l}\text { Apresentou algum problema com seu trabalho ou atividades habituais } \\
\text { devido à sua saúde física nos últimos } 60 \text { dias }\end{array}$} \\
\hline Não & 118 & 78,1 \\
\hline Sim & 33 & 21,9 \\
\hline \multicolumn{3}{|c|}{$\begin{array}{l}\text { Os problemas com sua saúde física ou emocional interferiram com } \\
\text { atividades sociais }\end{array}$} \\
\hline Não & 78 & 51,7 \\
\hline Sim & 73 & 48,3 \\
\hline \multicolumn{3}{|l|}{ Você sente dor física } \\
\hline Não & 69 & 45,7 \\
\hline $\operatorname{Sim}$ & 82 & 54,3 \\
\hline \multicolumn{3}{|l|}{ Quando sente dor física, atrapalha a sua rotina } \\
\hline Não & 60 & 39,7 \\
\hline Sim & 49 & 32,5 \\
\hline Não sabe & 42 & 27,8 \\
\hline \multicolumn{3}{|l|}{ Percepção em relação à doença renal crônica e ao tratamento dialítico } \\
\hline Positiva & 107 & 70,9 \\
\hline Negativa & 44 & 29,1 \\
\hline \multicolumn{3}{|c|}{$\begin{array}{l}\text { Em relação aos efeitos da doença renal crônica e ao tratamento } \\
\text { dialítico, em quais pontos a doença lhe incomoda }\end{array}$} \\
\hline Mudanças nos hábitos diários (dietética, hídrica e laboral) & 119 & 78,8 \\
\hline Dificuldade de viajar & 6 & 4,0 \\
\hline Necessidade de serviços médicos e outros profissionais de saúde & 7 & 4,6 \\
\hline Exposição ao estresse e preocupações com a doença e tratamento & 3 & 2,0 \\
\hline Não sabe & 16 & 10,6 \\
\hline
\end{tabular}

\section{DISCUSSÃO}

O número de pessoas com doença renal crônica vem aumentando gradativamente no Brasil e no mundo podendo estar relacionado com o aumento da expectativa de vida da população, da incidência e prevalência das doenças crônicas tais como hipertensão arterial, diabetes mellitus e doenças cardiovasculares, que são apontadas como as principais doenças de base dos portadores de doença renal crônica. ${ }^{1,3-4}$

0 perfil sociodemográfico identificado neste estudo corrobora dados evidenciados em outros estudos científicos, ${ }^{8,10-1}$ entretanto, ressalta-se que fatores como o predomínio de idosos, sexo masculino, baixa escolaridade e fonte de renda baixa vinculada à aposentadoria e ao auxílio-doença acentuam a vulnerabilidade a qual estes indivíduos já estão expostos por conta da doença renal crônica. Desse modo, torna-se necessário o fortalecimento da rede de suporte social, entre familiares e, em especial, com os diferentes serviços da rede de atenção à saúde, uma vez que a maioria dos sujeitos em tratamento utiliza transportes fornecidos por vários municípios da região. Com isso, existe uma limitação da participação do acompanhante durante esse processo.

Destaca-se, ainda, diante do perfil vulnerável, a importância do financiamento do Sistema Único de Saúde nos custos do tratamento da hemodiálise pela limitação dos portadores de doença renal crônica em manter-se em atividade profissional. Salientase que a difícil inserção no mercado de trabalho, após o início do tratamento, pode estar relacionada às dificuldades referidas pelos pacientes de alterações na imagem, do tempo gasto na hemodiálise e das condições físicas diminuídas para a realização das atividades laborais. ${ }^{12}$ Entretanto, no estudo, denota-se um número significativo de indivíduos que permanecem ativos no mercado de trabalho, mas que sobrevivem com até dois salários mínimos. Além disso, quase metade dos participantes referiu gastos com medicamentos, o que pode ocorrer em decorrência de algumas comorbidades coexistentes e especificidades da condição clínica relacionadas à resistência do organismo ao fármaco oferecido pela rede.

Ao considerar a assistência integral fundamental para o cuidado humano, é necessário destacar a importância da acessibilidade ao tratamento conservador e pré-diálise, que objetiva aumentar a sobrevida de pacientes que necessitam dessa terapêutica, bem como obter os parâmetros clínicos adequados. ${ }^{13}$ Neste estudo, a maioria teve cuidados pré-diálise por mais de seis meses e foi orientada sobre todas as modalidades de terapia de substituição renal. Não obstante, em muitos deles, o tratamento conservador foi inobservável, o que evidencia uma lacuna no acesso à saúde e nas ações de 
prevenção e manejo das condições crônicas, em especial, relacionada aos elementos modificáveis que são essenciais para postergar a progressão da doença.

Nesse cenário, a Enfermagem é muito presente no acompanhamento desses indivíduos a fim de controlar as manifestações clínicas na fase pré-dialítica e proporcionar, aos pacientes e familiares, esclarecimentos sobre as modalidades dialíticas, complicações e prevenção dos fatores de riscos modificáveis, controle das comorbidades existentes e redução dos comportamentos de risco relacionados à alimentação, sedentarismo, etilismo e tabagismo. Essa conduta favorece o esclarecimento a respeito dos possíveis agravos, o que potencializa sentimentos positivos de enfrentamento frente à doença e ao tratamento. ${ }^{9}$

Diante da complexidade do cuidado ao indivíduo com doença crônica, o funcionamento adequado e integrado da rede de atenção à saúde é essencial. Assim, o acompanhamento de todos os pacientes, pela equipe da Atenção Primária, favorece a organização da demanda com relação às reais necessidades. Além disso, no caso da doença renal crônica, possibilita o encaminhamento no momento adequado para o nefrologista, assim como a contrarreferência para a Unidade Básica de Saúde com vistas à manutenção do tratamento no âmbito domiciliar, à diminuição de internações hospitalares recorrentes e na prevenção de complicações que podem levar à mortalidade. $^{2}$

Apesar de grande parte dos participantes considerar boa sua autopercepção de saúde, apresenta sentimentos positivos frente às expectativas quanto à doença renal crônica e ao tratamento dialítico e acredita que a situação de saúde física e emocional não interfere em suas atividades sociais e rotina de vida. Muitos afirmaram que mudanças nos hábitos diários, como a dietética, a hídrica e a laboral, lhes incomodavam, o que mostra que essas mudanças alimentares, limitações nas atividades físicas, lazer, trabalho, a utilização contínua de medicações, assim como a dependência de familiares e de profissionais de saúde podem tornar a vida um grande desafio. ${ }^{14-5}$ Além disso, a restrição física de um dos braços, em decorrência da fístula arteriovenosa, o desconforto causado pelo cateter, quando é o caso, e a dor que foi relatada com frequência pelos pacientes deste estudo provocam mudanças no desempenho das atividades diárias e profissionais dos pacientes tornando-os mais inseguros no cuidado consigo mesmos. ${ }^{9}$
Este estudo teve como limitação ser realizado em um único centro de diálise que sofre influência das características peculiares da região.

\section{CONCLUSÃO}

Ao identificar a acessibilidade ao tratamento e o estado de saúde de pacientes em hemodiálise junto a elementos influentes no cuidado, conclui-se a necessidade do fortalecimento da rede de apoio social e de atenção à saúde, uma vez que a condição de vulnerabilidade em decorrência do estado de adoecimento é agravada por características como a baixa escolaridade, renda e idade avançada.

Além disso, elucidam-se novos desafios aos diferentes pontos de atenção à saúde, haja vista que a prevalência da inobservância de tratamento conservador reflete a insuficiência no desenvolvimento das ações de manejo da doença renal crônica na promoção à saúde, prevenção de complicações e até mesmo na acessibilidade e organização do fluxo de atendimento.

Os dados deste estudo fornecem subsídios para que se repensem as políticas de atendimento, o papel dos profissionais de saúde e a importância do fortalecimento da rede de atenção perante a acessibilidade ao tratamento e o estado de saúde do paciente dialítico. Reitera-se a necessidade de incorporar as características encontradas, no planejamento de estratégias de cuidado, a fim de responder às reais necessidades identificadas. Por fim, a efetividade do funcionamento do sistema de referência e contrarreferência utilizada pelos diferentes pontos de atenção da rede oferecerá suporte para um cuidado integral e continuado a esses indivíduos.

\section{REFERÊNCIAS}

1. Inker LA, Astor BC, Fox CH, Isakova $\mathrm{T}$, Lash JP, Peralta CA, et al. KDOQI US commentary on the 2012 KDIGO clinical practice guideline for the evaluation and management of CKD. Am J Kidney Dis. 2014; 63(5):712-35. Doi: http://dx.doi.org/10.1053/j.ajkd.2014.01.416

2. Ministério da Saúde (BR). Secretaria de Atenção à Saúde. Departamento de Atenção Especializada e Temática. Diretrizes Clínicas para o Cuidado ao paciente com Doença Renal Crônica - DRC no Sistema Único de Saúde/ Ministério da Saúde. Secretaria de Atenção à Saúde. Departamento de Atenção Especializada e Temática. - Brasília: Ministério da Saúde, 2014. 
3. Liu FX, Rutherford P, Tomic KS, Prichard S, Laplante S. A global overview of renal registries: a systematic review. BMC Nephrology. 2015;16(31):1-10. Doi: https: / /doi.org/10.1186/s12882-015-0028-2

4. Sesso RC, Lopes AA, Thomé FS, Lugon JR, Martins CT. Brazilian Chronic Dialysis Census 2014. J Bras Nefrol. 2016; 38(1):54-61. Doi: https: //dx.doi.org/10.5935/0101-

\section{$\underline{2800.20160009}$}

5. Menezes FG, Barreto DV, Abreu RM, Roveda F, Pecoits-Filho RFS. Overview of hemodialysis treatment funded by the Brazilian Unified Health System: an economic perspective. J Bras Nefrol. 2015; 37(3):367-78. Doi: http: / /dx.doi.org/10.5935/0101-

2800.20150057

6. Alves LO, Guedes CCP, Costa BG. Nurses actions for chronic renal patients: reflection of comprehensive care focus. J Res Fundam Care Online. 2016; 8(1):3907-21. Doi: http://dx.doi.org/10.9789/2175-

5361.2016.v8i1.3907-3921

7. Silva RAR, Souza VL, Oliveira GJN, Silva BCO, Rocha CCT, Holanda JRR. Coping strategies used by chronic renal failure patients on hemodialysis. Esc. Anna Nery. 2016; 20(1):147-54. Doi: http: / /dx.doi.org/10.5935/1414-

\subsection{0}

8. Gesualdo GD, Zazzetta MS, Say KG, Orlandi FS. Factors associated with the frailty of elderly people with chronic kidney disease on hemodialysis. Ciênc. saúde coletiva [online]. 2016; 21(11):3493-98. Doi: http://dx.doi.org/10.1590/1413-

\subsection{5}

9. Spigolon DN, de Souza JP, da Silva CA, Zollner SDL, Modesto AP. Perception on health and disease, patient in treatment on hemodialysis: can feeling healthy be enough? Ann Nurs Pract [Internet]. 2016 [cited 2016 Out 18];3(6):1063. Available from: https: //www.jscimedcentral.com/Nursing/nu rsing-3-1063.pdf

10. AlDukhayel A. Prevalence of depressive symptoms among hemodialysis and peritoneal dialysis patients. Int J Health Sci (Qassim) [Internet]. 2015 Jan [cited 2016 Out 18];9(1):9-16. Available from: https: //www.ncbi.nlm.nih.gov/pmc/articles/ PMC4394933/

11. Telles CT, Dobner T, Pomatti G, Fortes VF, Brock F, Bettinelli LA. Socio-demographic, clinical and laboratory profile of patients submitted to hemodialysis. Rev Rene. 2014; 15(3):420-6. Doi: 10.15253/2175$\underline{6783.2014000300006}$
12. Silva G, Nóbrega JYL, Nóbrega AL, Araújo RLD, Silva NQ, Nóbrega MF, et al. Percepção de portadores de doença renal crônica com relação ao tratamento hemodialítico. INTESA [Internet]. 2015 [cited 2016 Out 20];9(1):2330. Available from: http://www.gvaa.com.br/revista/index.p hp/INTESA/article/viewFile/3173/2849

13. Tsutsui $H$, Nomura $K$, Ishiguro A, Tsuruta Y, Kato S, Ysuda $Y$, et al. Factors associated with employment in patients undergoing hemodialysis: a mixed methods study. Renal Replacement Therapy. 2017; 3(23):2-11. Doi: https://doi.org/10.1186/s41100-017-0105-z

14. Spigolon DN, de Moraes TP, Figueiredo AE, Modesto AP, Barretti P, Bastos MG, et al. Impact of pre-dialysis care on clinical outcomes in peritoneal dialysis patients. Am J Nephrol. 2016; 43(2):104-11. Doi: http://dx.doi.org/10.1159/000444401

15. Fortes VLF, Bettinelli LA, Pomatti DM, Brock J, Dobner T. The chronic renal disease course: from early symptons to discovery. Rev Rene [Internet]. 2013 [cited 2016 Out 18];14(3):531-40. Available from: file:///C:/Users/Lenovo/Downloads/34286423-1-SM.pdf

Submissão: 31/01/2018

Aceito: 18/05/2018

Publicado: 01/07/2018

\section{Correspondência}

Edilaine Maran

Universidade Estadual do Paraná

Av. Gabriel Esperidião - Parque Morumbi CEP: 87703-000 - Paranavaí (PR), Brasil 